\title{
Efficient Modeling of the Wireless Power Transfer Efficiency for Varying Positions and Orientations between Transmitter and Receiver
}

\author{
Gert-Jan Stockman, Dries Vande Ginste and Hendrik Rogier \\ IBCN/Electromagnetics Group, Department of Information Technology, Ghent University/iMinds
}

\begin{abstract}
A method that efficiently calculates the Power Transfer Efficiency (PTE) of a Wireless Power Transfer (WPT) system is described in this paper. It allows for arbitrary relative positions and orientations between devices in the system, both in far-field and radiative near-field configurations. The method uses a single simulation or measurement of the radiation patterns of the antennas employed in the WPT system, from which the interaction between devices at any relative position and orientation can be modeled. A spherical harmonics decomposition, together with Wigner-D rotation matrices, is applied to perform efficient translations and rotations of the devices used in the WPT system.
\end{abstract}

Index Terms-Power Transfer Efficiency, Radiative NearField, Spherical Harmonics, Wigner D-Matrix, Wireless Power Transfer.

\section{INTRODUCTION}

Near-field Wireless Power Transfer (WPT) has gained more interest over the years due to its use in Radio Frequency Identification (RFID) systems [1]-[3]. In this paper, we discuss a method to efficiently model the interaction between devices in the radiative near-field. In particular, it is shown that the electromagnetic interaction between devices in a WPT system with arbitrary position and orientation can be computed using a single simulation or measurement of the individual radiation patterns of these devices. This information is sufficient to model the Power Transfer Efficiency (PTE) in a realistic WPT system in the radiative near-field (and beyond) with high accuracy, for different possible configurations of the devices in the system. A meaningful application example illustrates the outlined theory.

In the sequel, all sources and fields are assumed to be time harmonic with angular frequency $\omega$ and with time dependencies $e^{j \omega t}$ suppressed. Unit vectors are denoted with a "hat", e.g. $\hat{\mathbf{v}}$.

\section{FORMALISM}

Consider two antennas, a transmitter TX and a receiver RX, with radiation patterns $\mathbf{F}_{\mathrm{TX}}(\hat{\mathbf{k}})$ and $\mathbf{F}_{\mathrm{RX}}(\hat{\mathbf{k}})$, respectively, where $\hat{\mathbf{k}}=\sin \theta \cos \phi \hat{\mathbf{x}}+\sin \theta \sin \phi \hat{\mathbf{y}}+\cos \theta \hat{\mathbf{z}}$. Then it was shown in [4] that, in the radiative near-field, the short-circuit current $I_{s c}$ at the terminals of the receiver can be described as:

$$
I_{s c}=-\frac{1}{Z V_{0}} \int_{\Omega} T\left(\mathbf{r}_{\mathrm{TX}, \mathrm{RX}}, \hat{\mathbf{k}}\right) \mathbf{F}_{\mathrm{TX}}(\hat{\mathbf{k}}) \cdot \mathbf{F}_{\mathrm{RX}}(-\hat{\mathbf{k}}) \mathrm{d} \hat{\mathbf{k}} .
$$

Here, we integrate over the Ewald sphere $\Omega$. Furthermore, $V_{0}$ is a normalization factor, $Z$ the wave impedance of the background medium, $\mathbf{r}_{\mathrm{TX}, \mathrm{RX}}$ the vector between the phase center of the receiver and the phase center of the transmitter, and $T\left(\mathbf{r}_{\mathrm{TX}, \mathrm{RX}}, \hat{\mathbf{k}}\right)$ the translation operator. Expression (1) is valid as long as the two antennas (devices) are not positioned in each other's reactive near-field. We will now extend the above result into a formalism that is of practical use for the calculation of the PTE in a WPT system. Thereto, first, we want to be able to efficiently turn the antennas about their phase center. This can be done by applying the appropriate rotation $\mathcal{R}$ to the radiation patterns of the devices. The rotation is efficiently performed in the spherical harmonics domain $\left(\mathcal{R}_{S H}\right)$, as shown in Fig 1 . The transformation to the

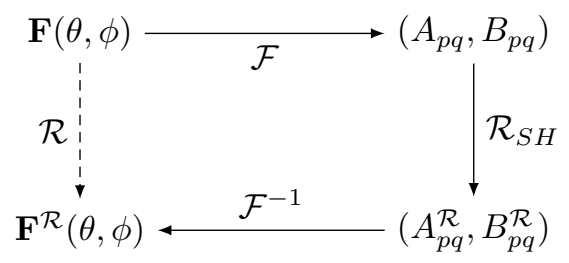

Fig. 1: Rotation of $\mathbf{F}(\theta, \phi)$ to $\mathbf{F}^{\mathcal{R}}(\theta, \phi)$ using the spherical harmonics domain.

spherical harmonics domain $\mathcal{F}$ and its inverse $\mathcal{F}^{-1}$ are given by (2) and (3), respectively:

$$
\begin{gathered}
\left\{\begin{array}{c}
A_{p q} \\
B_{p q}
\end{array}\right\}=-\frac{1}{p(p+1)} \int_{\phi=0}^{2 \pi} \int_{\theta=0}^{\pi}\left[q\left\{\begin{array}{c}
j F_{\phi}(\theta, \phi) \\
-F_{\theta}(\theta, \phi)
\end{array}\right\} Y_{p q}^{*}(\theta, \phi)\right. \\
\left.+\sin \theta\left\{\begin{array}{c}
-F_{\theta}(\theta, \phi) \\
j F_{\phi}(\theta, \phi)
\end{array}\right\} \frac{\mathrm{d} Y_{p q}^{*}(\theta, \phi)}{\mathrm{d} \theta}\right] \mathrm{d} \theta \mathrm{d} \phi \\
\left\{\begin{array}{c}
F_{\theta}(\theta, \phi) \\
F_{\phi}(\theta, \phi)
\end{array}\right\}=\sum_{p=0}^{P} \sum_{|q| \leq p}\left[\left\{\begin{array}{c}
A_{p q} \\
j B_{p q}
\end{array}\right\} \frac{\mathrm{d} Y_{p q}(\theta, \phi)}{\mathrm{d} \theta}\right. \\
\left.+\left\{\begin{array}{c}
B_{p q} \\
j A_{p q}
\end{array}\right\} \frac{q Y_{p q}(\theta, \phi)}{\sin \theta}\right]
\end{gathered}
$$

Here, $Y_{p q}(\theta, \phi)$ are the orthonormalized scalar spherical harmonics and $P$ is a parameter that determines the accuracy.

In Fig. 1, the transformation $\mathcal{R}$ represents the desired rotation in the spatial domain. The rotation itself is performed in the spherical harmonics domain by the transformation $\mathcal{R}_{S H}$ 

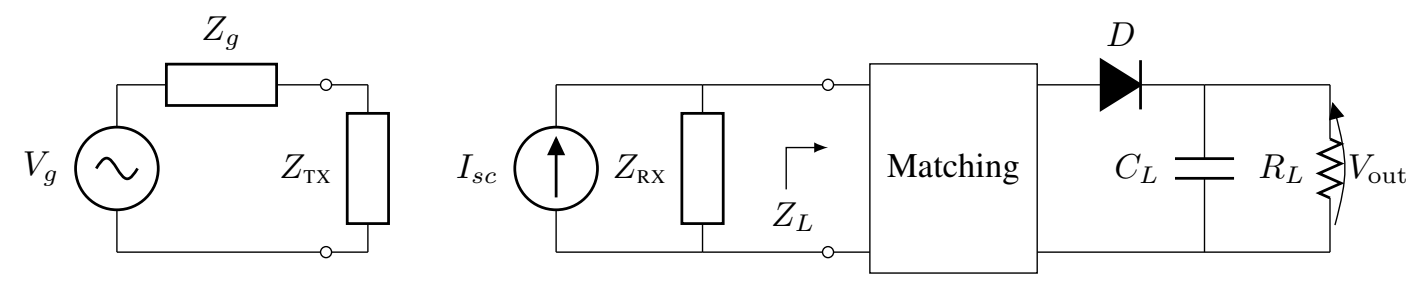

Fig. 2: Equivalent circuit of a WPT link, i.e. a transmit antenna and a receive antenna with rectifier (rectenna).

that makes use of Wigner D-matrices. The rotated coefficients of the spherical harmonics expansion are calculated as [5]

$$
\left\{\begin{array}{l}
A_{p q}^{\mathcal{R}} \\
B_{p q}^{\mathcal{R}}
\end{array}\right\}=\left\{\begin{array}{l}
A_{p q} \\
B_{p q}
\end{array}\right\} \sum_{|r| \leq p} e^{-j q \gamma} d_{p q}^{r}(\beta) e^{-j r \alpha},
$$

with $d_{p q}^{r}(\beta)$ the Wigner small d-matrix, given by [6]

$$
\begin{aligned}
d_{p q}^{r}(\beta)= & (-1)^{r-q} \sqrt{(p+r) !(p-r) !(p+q) !(p-q) !} \\
& \cdot \sum_{s}(-1)^{s} \frac{\left(\cos \frac{\beta}{2}\right)^{2(p-s)+q-r}\left(\sin \frac{\beta}{2}\right)^{2 s-q+r}}{(p+q-s) ! s !(r-q+s) !(p-r-s) !} .
\end{aligned}
$$

Here, the range of $s$ is determined by the condition that all factorials are nonnegative, thus $s \in$ $[\max (0, q-r), \min (p+q, p-r)]$. In (4)-(5), $\alpha, \beta$ and $\gamma$ are the standard Euler angles that define the rotation using the $z-y-z$ convention in a right-handed frame. The Euler angles $(\alpha, \beta, \gamma)$ are readily related to the desired inclination and azimuthal angles $\theta$ and $\phi$, by choosing $\alpha=\phi, \beta=\theta$ and $\gamma=0$.

Second, the rotation mechanism of Fig. 1 can be combined with a translation, by simply changing $\mathbf{r}_{\mathrm{TX}, \mathrm{RX}}$ in the translation operator $T\left(\mathbf{r}_{\mathrm{TX}, \mathrm{RX}}, \hat{\mathbf{k}}\right)$ in (1). Consequently, we are now able to calculate the wireless link efficiency between devices in a WPT system, positioned anywhere in space, and with any relative orientation. To calculate the wireless link efficiency, we need the power delivered to the receiving antenna. Fig. 2 represents the equivalent circuit of a WPT link. The wireless link efficiency can now be written as

$$
\eta_{\text {link }}=\frac{P_{\mathrm{RX}}}{P_{\mathrm{TX}}}=\frac{\frac{1}{2} \Re\left(Z_{L}\left|I_{s c}\right|^{2}\left|\frac{Z_{\mathrm{RX}}}{Z_{\mathrm{Rx}}+Z_{L}}\right|^{2}\right)}{\frac{1}{2 Z} \int_{\Omega}\left|\mathbf{F}_{\mathrm{TX}}(\hat{\mathbf{k}})\right|^{2} \mathrm{~d} \hat{\mathbf{k}}},
$$

with the numerator representing the power delivered to the receiving antenna's load $Z_{L}$ and the denominator the power emitted by the (rotated) transmitter with (rotated) radiation pattern $\mathbf{F}_{\mathrm{TX}}(\hat{\mathbf{k}})$.

To extend the wireless link efficiency to the PTE, we need the ratio of Direct Current (DC) power dissipated in the load $R_{L}$ at the receiving side to the Alternating Current (AC) power delivered to the transmit antenna. Assuming no radiation losses, this AC power equals $P_{\mathrm{TX}}$. The DC power delivered to the load is found by combining the link efficiency calculated in (6) with the efficiency of the matching circuit and of the rectifier. Since the input impedance $Z_{L}$ of a nonlinear circuit depends on the incoming power, the efficiency of the matching network, denoted $\eta_{\text {match }}$, will also have this dependency. This matching efficiency can be simulated using commercial tools such as Advanced Design System (ADS). The efficiency of the rectifier circuit itself can be calculated by comparing the DC power, delivered to the load resistor $R_{L}$, to the incoming power $P_{\text {inc }}=\eta_{\text {match }} \cdot P_{\mathrm{RX}}$, injected into the rectifier circuit, i.e. $\eta_{\text {rect }}=P_{\text {inc }} / P_{\mathrm{DC}}$. The DC power $P_{\mathrm{DC}}$ delivered to the load $R_{L}$ is calculated as $P_{\mathrm{DC}}=V_{\text {out }}^{2} / R_{L}$ where the DC output voltage $V_{\text {out }}$ may also be found from simulation tools such as ADS. The PTE of the complete system is given by $\mathrm{PTE}=P_{\mathrm{DC}} / P_{\mathrm{TX}}=\eta_{\text {link }} \cdot \eta_{\text {match }} \cdot \eta_{\text {rect }}$.

\section{ApPlication EXAMPLE}

To demonstrate the practical applicability and the efficiency of our method, an application example is considered. As the transmitting device, we choose a Standard Gain Horn (SGH) antenna radiating at $1.5 \mathrm{GHz}$ with a power of $10 \mathrm{dBm}$. As the receiving device, an inset fed microstrip patch antenna, also designed to resonate at $1.5 \mathrm{GHz}$, is used. The antennas are placed at a separation distance $d=5 \lambda$ as shown in Fig. 3 . The patch antenna is rotated using the $z-y-z$ convention

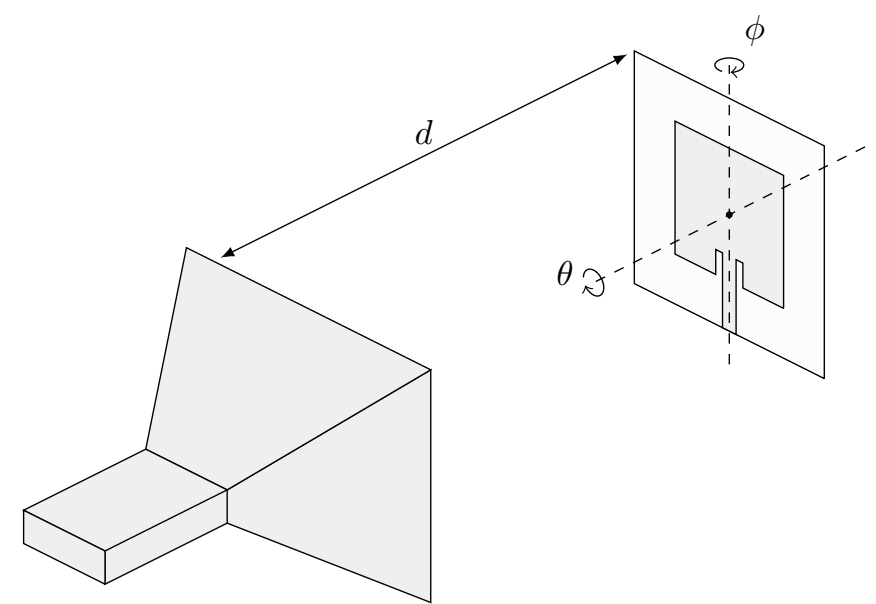

Fig. 3: Simulation setup where an SGH acts as transmitter and a patch antenna as receiver.

in a right-handed frame over $-\pi / 2 \leq \alpha=\phi \leq \pi / 2$, $-\pi / 2 \leq \beta=\theta \leq \pi / 2$ and $\gamma=0$ for a total of $91 \times 91$ points. At the receiving end, a rectifier was attached to the patch antenna to form a rectenna. This complete rectenna was designed in ADS and consists of a matching network, a voltage doubler and a rectifier as shown in Fig. 4. The antenna 


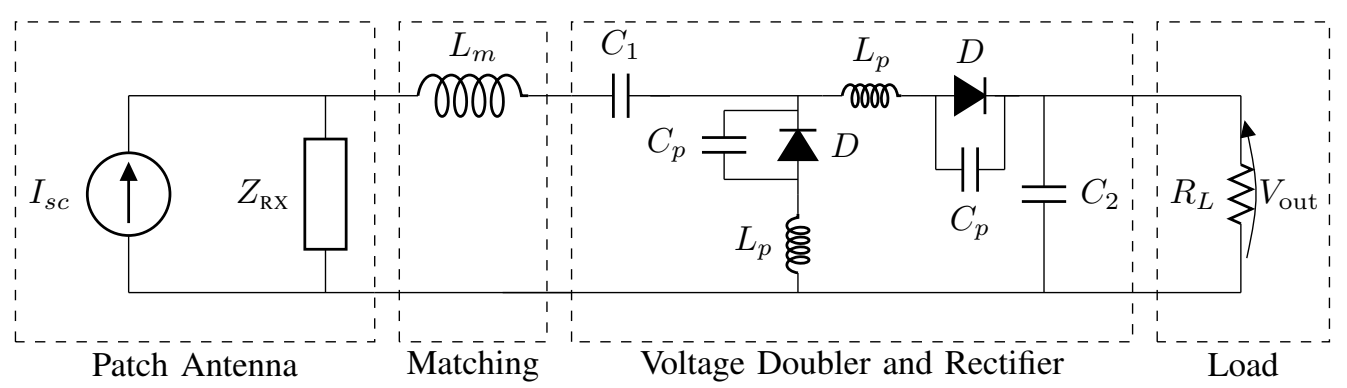

Fig. 4: The complete schematic of a rectenna element as designed and simulated in ADS.

and the voltage doubler and rectifier circuit are matched using an inductor $L_{m}=5 \mathrm{nH}$. The voltage doubler and rectifier circuit itself consists of two HSMS-2850 Schottky diodes together with their package parasitics $C_{p}=0.08 \mathrm{pF}$ and $L_{p}=2 \mathrm{nH}$. For these diodes a pertinent SPICE model was used. The capacitors used are $C_{1}=C_{2}=100 \mathrm{pF}$ and this example was designed for a load resistance of $R_{L}=100 \Omega$. The matching circuit was designed to have optimal matching when $P_{\mathrm{RX}}=-10 \mathrm{dBm}$. Since the input impedance of the voltage doubler and rectifier circuit depends on incoming power, due to the nonlinear diodes, the matching efficiency $\eta_{\text {match }}$ also depends on the incoming power $P_{\mathrm{RX}}$. Using a harmonic balance simulation in ADS, the input impedance of the voltage doubler and rectifier circuit is calculated for different incoming powers, from which the matching efficiency is then calculated as

$$
\eta_{\text {match }}=1-\left|\frac{Z_{L}\left(P_{\mathrm{RX}}\right)-Z_{\mathrm{RX}}^{*}}{Z_{L}\left(P_{\mathrm{RX}}\right)+Z_{\mathrm{RX}}}\right|^{2} .
$$

Here, $Z_{L}\left(P_{\mathrm{RX}}\right)$ is the input impedance of the matching circuit, depending on the incoming power to the matching circuit. Furthermore, the efficiency of the voltage doubler and rectifier circuit $\eta_{\text {rect }}$ is simply calculated by dividing the DC power delivered to the load by the AC power delivered to the input of this circuit. Subsequently, these efficiencies can be combined with the wireless link efficiency $\eta_{\text {link }}$ to obtain the complete PTE over all angles, by substituting the appropriate rotated radiation patterns of the devices in (1). Here, the radiation pattern of the SGH is calculated analytically and the radiation pattern of the patch antenna is obtained using ADS. The rotations are performed using the spherical harmonics domain as explained above with $P=10$. The resulting PTE for all $91 \times 91$ angles is given in Fig. 5. The average simulation time per sample is $92 \mathrm{~ms}$. All simulations have been carried out on an Intel@ Core $^{\mathrm{TM}}$ i7-2600 processor running at $3.40 \mathrm{GHz}$ and with $16 \mathrm{~GB}$ of memory.

\section{CONCLUSION}

We have proposed a method to efficiently predict the PTE of a complete WPT system in the radiative near-field. The method relies on a single measurement or simulation of the radiation patterns of the devices in the system. The formalism explained in this contribution, allows to account

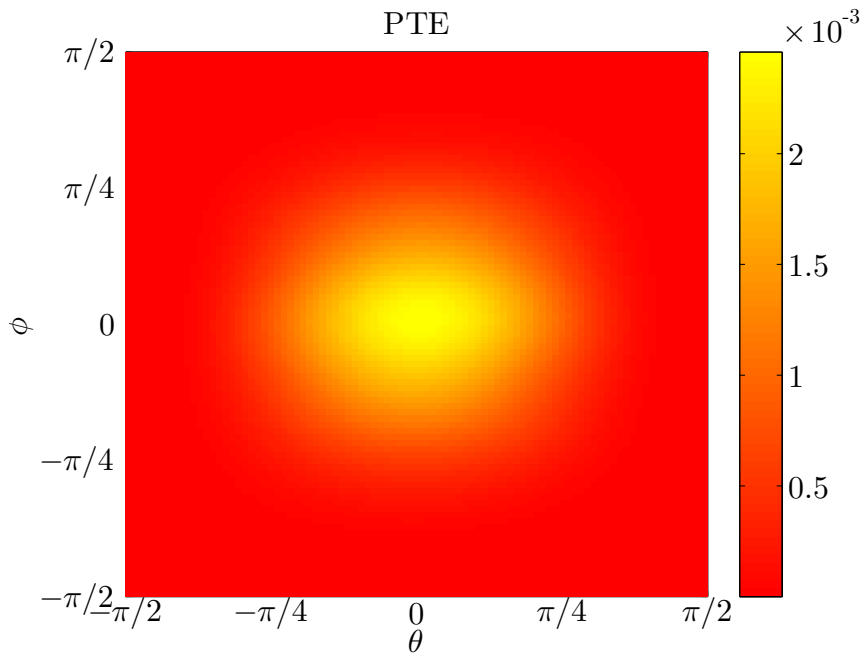

Fig. 5: PTE of the complete WPT system calculated for $-\pi / 2 \leq \phi \leq \pi / 2$ and $-\pi / 2 \leq \theta \leq \pi / 2$ and a separation distance $d=5 \lambda$.

for any relative position or orientation of the devices without requiring a new simulation for every alteration.

\section{REFERENCES}

[1] A. Buffi, P. Nepa, and G. Manara, "Analysis of near-field coupling in UHF-RFID systems," in 2011 IEEE-APS Topical Conference on Antennas and Propagation in Wireless Communications (APWC), Sept 2011, pp. 931-934.

[2] J. Lee and S. Nam, "Fundamental aspects of near-field coupling small antennas for wireless power transfer," IEEE Transactions on Antennas and Propagation, vol. 58, no. 11, pp. 3442-3449, Nov 2010.

[3] H. Visser and R. Vullers, "RF energy harvesting and transport for wireless sensor network applications: Principles and requirements," Proceedings of the IEEE, vol. 101, no. 6, pp. 1410-1423, June 2013.

[4] G.-J. Stockman, H. Rogier, and D. Vande Ginste, "Efficient modeling of interactions between radiating devices with arbitrary relative positions and orientations," IEEE Transactions on Electromagnetic Compatibility, vol. 56, no. 6, pp. 1313-1321, December 2014.

[5] R. Hoover, A. Maciejewski, and R. Roberts, "Pose detection of 3-D objects using images sampled on $\mathrm{SO}(3)$, spherical harmonics, and Wigner-D matrices," in IEEE International Conference on Automation Science and Engineering, CASE., Arlington, Virginia, 23-26 August 2008, pp. 47-52.

[6] Z. Gimbutas and L. Greengard, "A fast and stable method for rotating spherical harmonic expansions," Journal of Computational Physics, vol. 228, pp. 5621-5627, May 2009. 